\title{
The art and science of naming drugs
}

\section{Y} ou shuffle to the pharmacy counter, arthritic knees aflame, each step pure agony, and hand over a prescription for Cerebyx. Big mistake. Cerebyx is an anticonvulsant, not an anti-inflammatory. What you need is Celexa. Actually, no, that's an antidepressant. Instead, try ... Celebrex?

It's all so contusing — er, confusing. Why do so many drugs have such similar names? Well, there are, in fact, many reasons.

Coming up with a proprietary name for a new pharmaceutical product is no easy task. It shouldn't sound like that of another drug, according to health regulators, but with tens of thousands of pharmaceutical trademarks already on the books, it sometimes happens. Considering the other naming restrictions faced by drug companies - and the fact that, at last count, there were still only 26 letters in the alphabet the challenge of coming up with a distinctive, memorable, snappy name becomes apparent.

Pharmaceutical companies often pay outside agencies hundreds of thousands of dollars, even up to half a million, to develop a name for a product. The process starts several years before the company submits the drug to a health regulator to be approved for sale. Despite all that money and effort, however, many suggested drug names are still rejected by regulators such as the US Food and Drug Administration (FDA) and the European Medicines Agency.

"The general rules are: it can't sound or look like another drug product, which might result in a misprescription, and that's a safety issue; and the name can't make any [medical] claims or be overly promotional," says Scott Piergrossi, vice-president, creative, at the Brand Institute, a Florida-based company that has developed many brand names for drugs, including Levitra and Lipitor.

The name of a drug also can't serve to broaden indication, minimize risk or misleadingly imply uniqueness or superiority. For example, the FDA rejected the name NovoRapid (a type of insulin) because it implied it was fasteracting than its competitors; the name was changed to NovoLog. A name can't

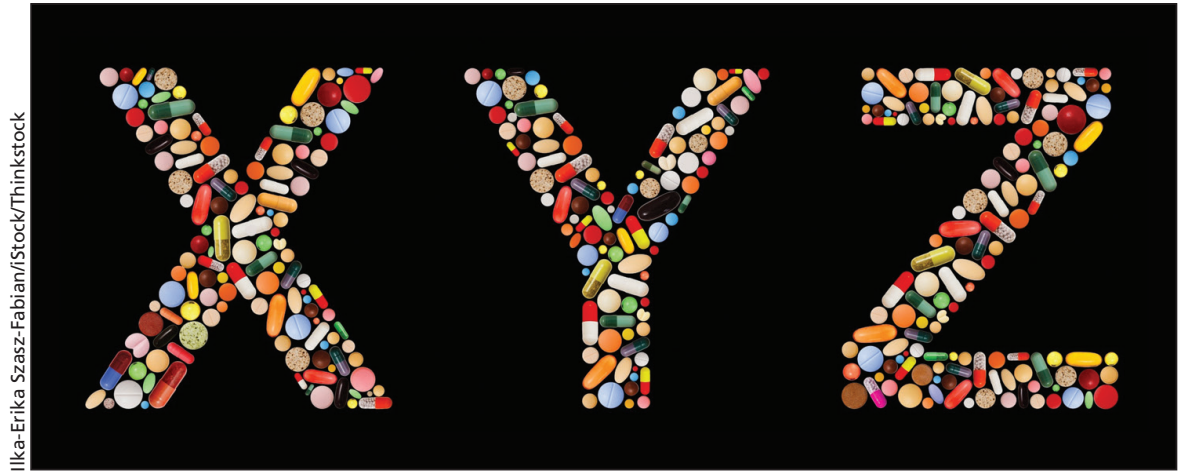

The letters " $\mathrm{X}$, " "Y" and "Z" often appear in drug brand names. Ever wonder why?

guarantee efficacy, either, which explains why the hair-regrowth product Rogaine couldn't be sold under the name Regain. A name can even be rejected merely for being too fanciful.

Then there are market considerations. If you fork over a half-mil for a name, after all, you want it to appeal to consumers. Pharmaceutical companies tend to favour names that are easy to pronounce in many languages and don't mean something offensive in another culture. They also try, despite all these restrictions, to use names that aren't entirely meaningless.

"When you are dealing with pharmaceutical names, they are largely coined names, so invented names, and they often try to encode, within the guidelines of not making claims or being overly promotional, some kind of message, whether it's aspirational or something that's functional pertaining to the efficacy of the product," says Piergrossi.

The name could, for instance, be associated with a positive word such as "victory" (the diabetes drug Victoza). It could be tied to a word implying what it does "levitate" and Levitra (erectile dysfunction) — or when it is used: "lune" (French for "moon") and Lunesta (insomnia).

The letters "X," "Y" and "Z" often appear in brand names because they give a drug a high-tech, sciency sounding name (Xanax, Xyrem, Zosyn). Conversely, "H," "J" and "W" are sometimes avoided because they are difficult to pronounce in some languages. Pharmaceutical products for women may include "S," "M" or " $L$ " to produce a softer sound (birth control pills Alesse, Yasmin, Seasonale).
The name of a drug may also reflect whether it is being primarily marketed to doctors or to the public. For general consumers, a name that is easy to say and remember is preferred. Physicians, on the other hand, may favour a name that hints at the chemical composition of the drug.

"In the pharmaceutical world, the brand names may not say very much to the layperson's eyes; a lot of the communication gets coded into two or three letters and placed as a prefix or as a suffix or as an infix, and the goal is that those letter strings can signal the science or some other message that is within reason, reflecting what it is the product does," says R. John Fidelino, executive director, creative, for InterbrandHealth, an agency headquartered in New York and behind popular pharmaceutical brand names such as Viagra and Prozac.

A good brand name, says Fidelino, can do much more than just move product; it can foster discussion and help reduce the stigma around certain medical conditions. Names that don't sound like scary chemicals stand a greater chance of entering the vernacular; people are more likely to talk about them and, in turn, the conditions they treat, be it mental illness, erectile dysfunction or some other tabooish malady.

"This is not a conversation about the esthetics of language," says Fidelino. "This is about the power of language to support proper understanding of the treatments that exist for serious medical conditions." - Roger Collier, CMAJ 